\title{
Hot-electron-assisted femtochemistry at surfaces: A time-dependent density functional
} theory approach

Gavnholt, Jeppe; Rubio, Angel; Olsen, Thomas; Thygesen, Kristian Sommer; Schiøtz, Jakob

Published in:

Physical Review B Condensed Matter

Link to article, DOI:

10.1103/PhysRevB.79.195405

Publication date:

2009

Document Version

Publisher's PDF, also known as Version of record

Link back to DTU Orbit

Citation (APA):

Gavnholt, J., Rubio, A., Olsen, T., Thygesen, K. S., \& Schiøtz, J. (2009). Hot-electron-assisted femtochemistry at surfaces: A time-dependent density functional theory approach. Physical Review B Condensed Matter, 79(19), 195405. https://doi.org/10.1103/PhysRevB.79.195405

\section{General rights}

Copyright and moral rights for the publications made accessible in the public portal are retained by the authors and/or other copyright owners and it is a condition of accessing publications that users recognise and abide by the legal requirements associated with these rights.

- Users may download and print one copy of any publication from the public portal for the purpose of private study or research.

- You may not further distribute the material or use it for any profit-making activity or commercial gain

- You may freely distribute the URL identifying the publication in the public portal 


\title{
gs \\ Hot-electron-assisted femtochemistry at surfaces: A time-dependent density functional theory approach
}

\author{
Jeppe Gavnholt, ${ }^{1}$ Angel Rubio, ${ }^{2}$ Thomas Olsen, ${ }^{1}$ Kristian S. Thygesen, ${ }^{3}$ and Jakob Schiøtz ${ }^{1, *}$ \\ ${ }^{1}$ Department of Physics, Danish National Research Foundation's Center for Individual Nanoparticle Functionality (CINF), \\ Technical University of Denmark, DK-2800 Kongens Lyngby, Denmark \\ ${ }^{2}$ Dpto. Fisica de Materiales, European Theoretical Spectroscopy Facility (ETSF), Universidad del Pais Vasco, \\ E-20018 Donostia-San Sebastian, Spain \\ ${ }^{3}$ Department of Physics, Center for Atomic-scale Materials Design (CAMD), Technical University of Denmark, \\ DK-2800 Kongens Lyngby, Denmark
}

(Received 15 January 2009; revised manuscript received 6 April 2009; published 6 May 2009)

\begin{abstract}
Using time-evolution time-dependent density functional theory (TDDFT) within the adiabatic local-density approximation, we study the interactions between single electrons and molecular resonances at surfaces. Our system is a nitrogen molecule adsorbed on a ruthenium surface. The surface is modeled at two levels of approximation, first as a simple external potential and later as a 20 -atom cluster. We perform a number of calculations on an electron hitting the adsorbed molecule from inside the surface and establish a picture, where the resonance is being probed by the hot electron. This enables us to extract the position of the resonance energy through a fitting procedure. It is demonstrated that with the model we can extract several properties of the system, such as the presence of resonance peaks, the time electrons stay on the molecule before returning to the surface when hitting a molecular resonance and the lowering of the resonance energy due to an image charge effect. Finally we apply the TDDFT procedure to only consider the decay of molecular excitations and find that it agrees quite well with the width of the projected density of Kohn-Sham states.
\end{abstract}

DOI: 10.1103/PhysRevB.79.195405

PACS number(s): 31.15.ee, 73.20.Hb, 82.20.Gk

\section{INTRODUCTION}

In most chemical processes the intrinsic barriers are overcome due to the randomly directed thermal energy of the atomic cores. This sets some limitations on the possibility of controlling chemical reactions since the thermal energy will distribute itself among all degrees of freedom in the system; i.e., the energy cannot be directed toward, for example, splitting of a certain molecule or desorption of another. Furthermore, in order to get a satisfactory turnover frequency, in some catalyzed reactions, the temperature may need to be so high that the catalyst becomes unstable and degrades over time.

In hot-electron-assisted femtochemistry at surfaces ${ }^{1-8}$ the hot electrons (electrons with an energy significantly above the Fermi level) interact with molecular resonances, which gives rise to an electron-phonon coupling. This will initiate motion mainly in those vibrational modes where the coupling is high; i.e., it is possible to direct energy toward certain vibrational modes. This has been demonstrated in an experiment by Bonn et al., ${ }^{8}$ who were able to form carbon dioxide from carbon monoxide and oxygen on a ruthenium (0001) surface with the help of hot electrons. This is normally impossible because the carbon monoxide desorbs before the carbon dioxide formation when the temperature is raised. The effect is explained by the hot-electrons injecting energy into the vibrational modes of the adsorbed atomic oxygen so that the barrier forming carbon monoxide can be overcome at a lower temperature.

In most femtochemistry experiments the hot electrons are generated using a femtosecond laser pulse. Each pulse excites a lot of electrons in the metal surface. Due to the high electron density the electron-electron scattering thermalize the hot electrons very rapidly, on a femtosecond time scale, giving rise to an electronic temperature, which is much higher than the phonon temperature. The phonons and electrons will equilibriate much slower, on a picosecond time scale, i.e., for several picoseconds there are electrons present, which have sufficient energy to interact with otherwise unreachable molecular resonances. The high concentration of high-energy electrons even makes it possible to observe multielectron processes, such as desorption induced by multiple electronic transitions, which has been observed for a variety of systems. ${ }^{9}$ However, the thermal distribution of electrons does not make it possible to target a specific molecular resonance, in particular one cannot inject electrons into a highenergy resonance without also injecting them into lowerenergy resonances, if present.

Another approach to generate hot electrons, by the use of a metal-insulator-metal (MIM) junction, has been suggested by Gadzuk. ${ }^{7}$ If the insulating layer in the junction is sufficiently thin and a finite bias is applied, electrons will tunnel from near the Fermi level of the first metal into the other metal, where they can have an energy significantly above the Fermi level; i.e., they will be hot electrons. If the second metal layer is also very thin, these electrons will be able to reach the surface of the other metal and perhaps induce chemistry. The advantage of such a device is that it should, at least theoretically, be possible to target certain molecular resonances by tuning the energy of the hot electrons. The disadvantage being that it will probably not be possible to generate a high electron flux, i.e., only single-electron processes can be observed. Such a MIM device, where the first metal has been substituted by a highly doped silicon layer, has recently been created, ${ }^{10}$ and its ability to induce chemical reactions is currently being investigated. 
We have previously investigated the hot-electron interaction with different diatomic molecules on different transitionmetal surfaces, by applying an electron-phonon interaction model to potential-energy surfaces, which are obtained from the delta self-consistent field method. ${ }^{11,12}$ The purpose of this paper is to investigate a different approach, based on timeevolution time-dependent density functional theory (TDDFT), for modeling hot electrons interacting with molecular resonances. TDDFT provides, in principle, an exact framework to describe nonequilibrium processes as the ones relevant in femtochemistry and transport. The price one has to pay in TDDFT is that all correlation effects are embedded in an exchange and correlation kernel that should be nonlocal in space and time. However, most functional in use nowadays are not designed to cope with nonequilibrium situations but still it is common practice to apply local and semilocal functionals to those situations. Further work is needed in the development of nonlocal and frequency-dependent exchange-correlation functionals, which would have impact beyond the present studies. The hope is, however, that such an approach with a simple exchange-correlation functional can still give supplementary information about the occurring processes. Of specific interest are the cross section for exciting the resonance and the lifetime the molecular excitations, which are very important when considering the possibility of hot-electrons inducing chemistry. ${ }^{12}$ Another nice feature of the time-evolution approach is that it offers the possibility of simulating the entire event of one hot electron hitting a molecule, i.e., it offers a more physically intuitive picture. Finally it is also worth mentioning that TDDFT provides a multicomponent approach, ${ }^{13}$ where the electron and nuclei motion can be directly coupled. This provides the hope that the TDDFT approach presented here in the future can involve a direct calculation of the induced molecular motion. A related approach is TDDFT-based Ehrenfest dynamics, which has, e.g., recently been used to study the interaction of a hydrogen atom with a jellium cluster. ${ }^{14}$

In the following we will start by giving a description of how the time-evolution TDDFT calculations have been performed. After this we present the simulations of the entire event of a hot electron hitting a molecule at a surface. We start by considering a simple model system and then move on to a more realistic system. Finally we will investigate the lifetime of molecular excitations by starting time-evolution TDDFT calculations from the excited state. All the way through we will focus on nitrogen adsorbed on ruthenium, although the methods presented of course are general.

\section{METHOD}

The main type of calculation performed in this paper is a time-evolution TDDFT calculation, which we have done with the freely available OCTOPUS code. ${ }^{15,16}$ We use an adiabatic local-density approximation (ALDA) (Ref. 17) description of the exchange-correlation functional. Nonadiabatic effects and initial state dependence of the exchange-correlation functional are not accounted for by the simple local-density approximation (LDA)-type functional. However, this is not a serious drawback for the present work where we are more interested in getting a qualitative rather than a quantitative picture of the process of hot-electron-induced femtochemistry at metal surfaces. To describe core electrons we use norm-conserving pseudopotentials from the Fritz-Haber Institute $^{18}$ generated using the Troullier-Martins scheme. ${ }^{19}$ OCTOPUS uses a real-space grid to represent wave functions and densities. After some convergence tests we found that a grid spacing of $0.18 \AA$ gives sufficient accuracy. The KohnSham equations are propagated using a combination of the exponential midpoint rule ${ }^{20}$ and a Krylov subspace approximation to the exponential matrix operator. ${ }^{21}$ The optimal time step for the type of systems considered here was found to be $0.001 \mathrm{fs}$, so this time step has been used in all calculations presented in this paper.

The time-evolution TDDFT calculations have not been started from the ground state but still ground-state calculations have been used in the generation of the initial states as it will be clear from Secs. III and IV. Unless otherwise specified the ground-state calculations have been made using the OCTOPUS code with a LDA (Ref. 22) description of the exchange-correlation interactions to maintain consistency with the TDDFT calculations. In each cycle toward selfconsistency in the Kohn-Sham equations a Broyden mixing ${ }^{23}$ of the seven preceding densities is performed and the Hamiltonian is diagonalized iteratively using the conjugategradient method. The occupations of the Kohn-Sham states follow a Fermi-Dirac distribution with an electronic temperature of $0.1 \mathrm{eV}$, which is necessary in order to get convergence.

\section{EXCITING AN ADSORBED MOLECULE}

In this section we consider the entire event of a single electron hitting a molecule adsorbed at a surface. We will start from a situation where an electron is placed inside the surface and has a momentum toward the molecule sitting on the surface. This means that we do not consider the creation of the hot electron but the simulated situation is very similar to that found in the MIM device, where hot electrons come from inside the surface with a sufficiently low rate, such that the hot electrons do not affect each other. Naturally, we expect the interaction between the hot electron and the molecular resonance to depend on the starting state of the hot electron, so in order to get some information on this dependence we will start by considering a very simple system. After this we will go to a more realistic system, consisting of a nitrogen molecule adsorbed on a ruthenium cluster.

\section{A. Nitrogen on a fictitious surface}

In this section we consider a very simple representation of a molecule adsorbed on a surface. As the adsorbed molecule we use the diatomic nitrogen molecule and instead of representing the surface with a lot of individual atoms, we use a simple external potential. One could just use a step function but in order to avoid any spurious effects due to the hard edges, we make a fit to the Kohn-Sham potential of a ruthenium slab density functional theory (DFT) calculation, which is shown in Fig. 1. 


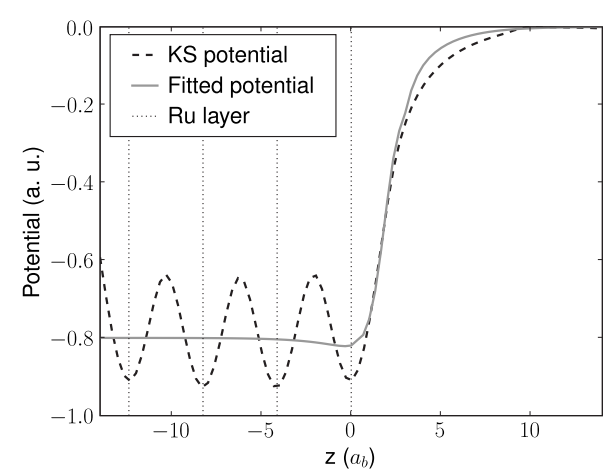

FIG. 1. A fit of the Kohn-Sham potential at a ruthenium surface. The dotted curve shows the self-consistent ground-state KohnSham potential of a four-layer ruthenium (0001) slab averaged over the directions parallel to the surface. The solid curve shows our fit from Eq. (1). The vertical lines indicate the positions of the layers in the slab. The DFT calculation was made with the GPAW code (Refs. 24 and 25).

The slab consists of four atomic layers and the exposed surfaces are close-packed (0001) surfaces. The shown KohnSham potential has been averaged over the directions parallel to the surface. The fitted potential is in atomic units ${ }^{26}$ (a.u.) given by the expression

$$
V_{\mathrm{fit}}(z)=-0.8 \frac{1}{1+e^{4(z-1)}}-0.16 e^{-|z-1.55|}
$$

where $z$ is the coordinate perpendicular to the surface and $z=0$ corresponds to the position of the outermost layer of atoms in the surface. The expression of Eq. (1) only represents the surface, i.e., the entire system of a surface and a molecule is handled by applying an external potential of $V_{\text {fit }}(z)+\Sigma_{a} V_{\text {cent }}^{a}(\mathbf{r})$, where $V_{\text {cent }}^{a}$ is the central potential of atom $a$ in the molecule. Furthermore the valence electrons of the molecule are included in the calculations, whereas no electrons of the surface are included.

This simple representation of the surface has the advantage that it is easy to generate a starting orbital for the hot electron inside the surface, which is orthogonal to the other occupied orbitals since all other electrons are located on the molecule outside the surface. Furthermore the computational effort is significantly lowered by the fact that only a few electrons are included in the calculation. Figure 2 shows an example of how the orbital of a hot electron evolves over time, when it starts inside the surface with a momentum toward the surface.

Before the time-evolution calculation the states of the valence electrons of the nitrogen molecule was found by performing a ground-state calculation with just these electrons. The starting orbital of the hot electron is an unoccupied eigenfunction of this ground-state Hamiltonian multiplied by $e^{i 0.8 z}$, in order to give it a momentum of 0.8 a.u. toward the surface. This eigenfunction was chosen because it is almost entirely located inside the surface and it has the $\Pi$ symmetry needed in order for it to interact with the $2 \pi^{*}$ states of the molecule.

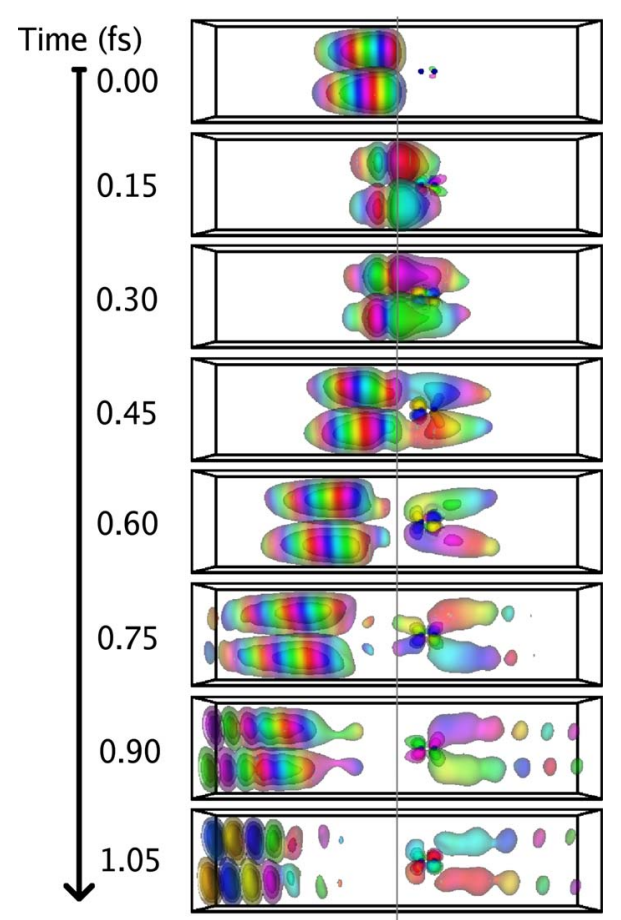

FIG. 2. (Color online) An example of the evolution over time of a hot electron with a momentum directed toward the molecule. The hot-electron orbital is shown at times: $0,0.15,0.30,0.45,0.60$, $0.75,0.90$, and $1.05 \mathrm{fs}$. The gray scale (online: color grading) indicates the phase of the orbital. The two dots, which are visible at $t$ $=0 \mathrm{fs}$, indicate the positions of the nitrogen atoms and the gray line indicates the surface. The unit cell is cylindrical with a radius of $4 \AA$ and a length of $40 \AA$ and is exactly contained in the shown boxes.

Figure 2 shows several interesting features. When hitting the surface a large fraction of the electron is reflected due to the work function of the surface but some of the electron ends up in the $2 \pi^{*}$ states of the molecule, indicating a nonzero probability of exciting the molecule. In this calculation some of the electron is apparently also transmitted by the molecule, which indicates that some of the electron has an energy above the vacuum level. From the figure it is also obvious that it does not make sense to continue the calculation much further since the reflections of the electron at the unphysical unit-cell edges start to interfere with the molecule after approximately 1 fs.

For the calculation displayed on Fig. 2 we chose the starting orbital of the hot electron rather randomly. In order to get an idea of how the obtained results vary with the starting orbital we have carried out calculations with the hot electron starting in a number of different starting orbitals as illustrated in Table I. Each of the orbitals shown in Table I has an average momentum in the $z$ direction of 0.8 a.u. but we also made several calculations on the same orbitals but with different average momenta, i.e., multiplied with a different exponential factor $\left(e^{i p_{0} z}\right)$. The unit cells have been made twice as long as the unit cell in Fig. 2 in order to prolong the time it takes before reflected waves reach the molecule.

The fraction of the electron that gets into the $2 \pi^{*}$ orbitals of the molecule varies a lot from calculation to calculation. 
TABLE I. (Color online) The five different types of wave functions we use as starting orbitals for the hot electron in Sec. III. The first order Bessel function used in the radial direction for wave functions $1-3$ is scaled such that the first node coincides with the unit-cell boundary. The gray scale (online: color grading) indicates the phase of the wave functions. The unit cells are cylindrical with a radius of $6 \AA$ and a length of $80 \AA$.

Wf. no. Picture Specifications

\section{Radial direction:}

1. order Bessel function
1

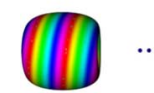

\section{z direction:}

Gaussian wavepacket with

$p_{0}=0.8, \Delta p=0.1$

\section{Radial direction:}

1. order Bessel function
2

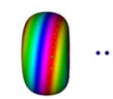

\section{z direction:}

Gaussian wavepacket with

$p_{0}=0.8, \Delta p=0.2$

\section{Radial direction:}

1. order Bessel function

\section{$\mathrm{z}$ direction:}

Gaussian wavepacket with

$p_{0}=0.8, \Delta p=0.4$

Eigenfunction for the

ground state Hamiltonian

4 D.

(eigenfunction no. 7)

times $e^{i p_{0} x}, p_{0}=0.8$

Eigenfunction for the

ground state Hamiltonian

$5 \quad 00$.

(eigenfunction no. 13)

times $e^{i p_{0} x}, p_{0}=0.8$
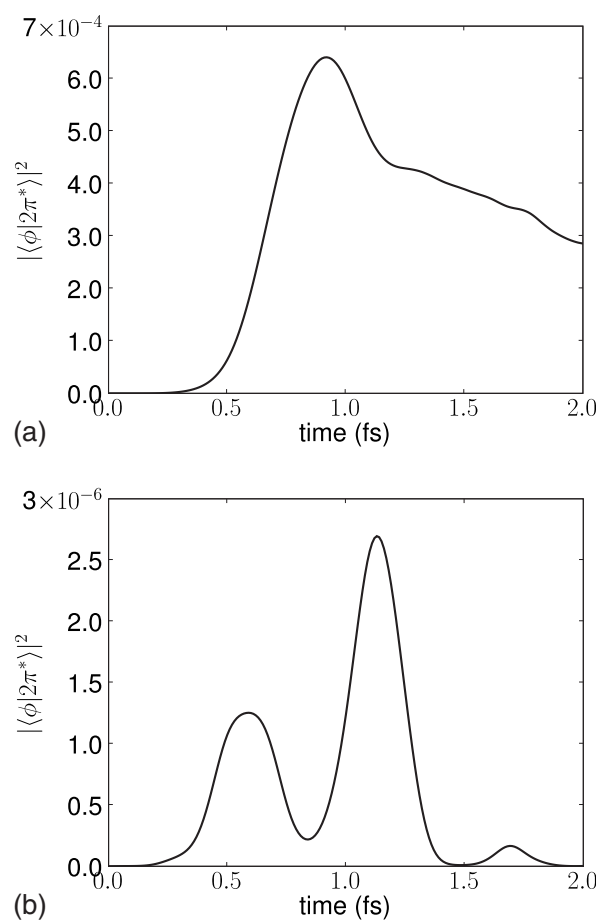

FIG. 3. The difference between an electron that hits resonance and one that does not. The $y$ axes are the orbital of the hot electron, $\phi$, projected to the subspace spanned by the two $2 \pi^{*}$ orbitals of the molecule squared. The $x$ axes show the time. Top panel is an example of an electron hitting resonance (Wf. No. 1 from Table I with $p_{0}=0.8$ ). Bottom panel is an example of an electron not hitting resonance (Wf. No. 1 from Table I with $p_{0}=0.4$ ). Please notice the more than 2 orders of magnitude difference in the $y$-axis scales.

The upper and lower panels in Fig. 3 illustrate the situations where a rather large part and a rather small part, respectively, of the electron goes into the $2 \pi^{*}$ orbitals. The $y$ axes show the projection of the orbital of the hot electron onto the subspace spanned by the two $2 \pi^{*}$ orbitals of the molecule and the $x$ axes are time. In the top panel the electron seems to hit resonance since a rather large part of it gets into the $2 \pi^{*}$ orbital. Furthermore it is seen that the excitation is quite long lived compared to the small fluctuations in the off-resonance calculation shown on the lower panel.

The results of all the calculations have been collected in Fig. 4, which shows the maximum overlap between the hotelectron orbital and the $2 \pi^{*}$ orbitals of the molecule squared, $\left|\left\langle\phi \mid 2 \pi^{*}\right\rangle\right|^{2}$, as a function of the average momentum for each of the five different starting orbitals from Table I. By maximum overlap we mean the maximum overlap within the first $2 \mathrm{fs}$, which is time enough for the wave function to fully hit the molecule but not enough time for the reflections at unitcell boundaries to interfere with the results. For all the curves there is a large dependence on the momentum, i.e., there are certain values of $p_{0}$ which are at resonance and others which are off-resonance. The curves are, however, also quite different. Curves 1-3 all have the maximum at the same momentum but it is also clear that the more well-defined momentum the wave function has, the more well defined is the resonance peak. This indicates that the shape of the resonance peaks are reflected by the Fourier transforms of the wave functions. 


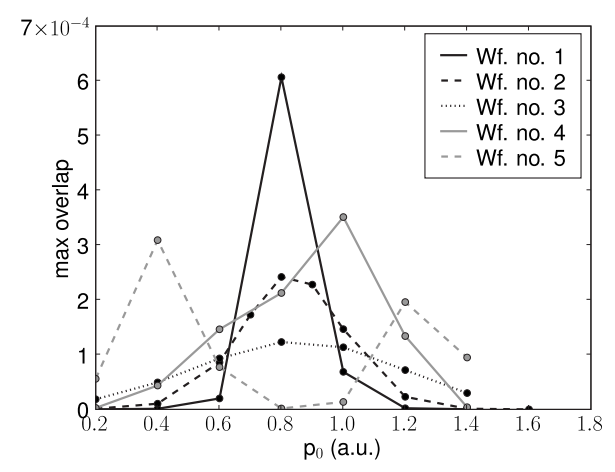

FIG. 4. The amount of electron that gets into the $2 \pi^{*}$ orbitals of the molecule within the first two femtoseconds plotted as a function of the average momentum of the hot electron, $p_{0}$. The five different curves are for the five different orbitals given in Table I.

This is supported by Fig. 5, which shows the Fourier transforms of wave functions 4 and 5 integrated over the axes parallel to the surface. These curves resemble the resonance curves on Fig. 4 a lot. One interpretation of this is that the wave function, which is sent toward the molecule, is just probing the resonance. In an energy picture this means that the maximal overlap, MO, which we interpret as the excitation probability, can be written as

$$
\mathrm{MO}=\int R(\epsilon) W(\epsilon) d \epsilon,
$$

where $W(\epsilon)$ is the amount of the hot electron that has the energy $\epsilon$, i.e.,

$$
W(\epsilon)=\sum_{i}\left|\left\langle\phi \mid \psi_{\mathrm{KS}}^{i}\right\rangle\right|^{2} \delta\left(\epsilon-\epsilon_{\mathrm{KS}}^{i}\right) .
$$

$\phi$ is the hot-electron orbital and $\psi_{\mathrm{KS}}^{\dot{ }}$ is the $i$ th Kohn-Sham orbital, which has the energy $\epsilon_{\mathrm{KS}}^{i} \cdot R(\epsilon)$ is the energy representation of the resonance. The difference of the Kohn-Sham eigenvalues do not describe excitation energies, therefore in Eq. (3) we are neglecting the renormalizaton of the KohnSham eigenvalues by the exchange-correlation kernel. For resonances as in the case of molecular systems in front of metallic surfaces this renormalization can be accounted for. ${ }^{27}$

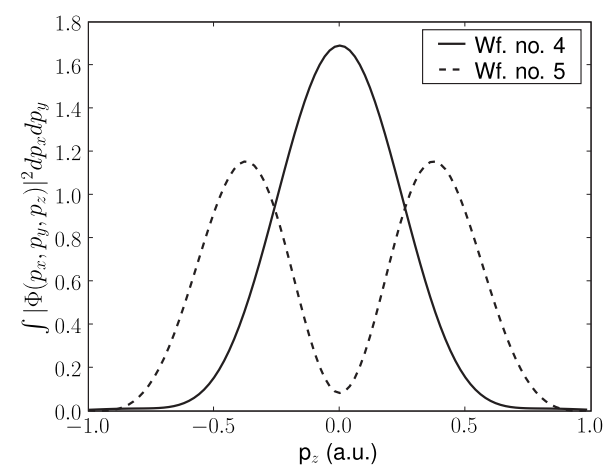

FIG. 5. The Fourier transform of wave functions 4 and 5 from Table I integrated over the momenta parallel to the surface. The horizontal axis indicates the momentum in the direction perpendicular to the surface, $p_{z}$.
For the resonance we will assume a Lorentzian shape,

$$
R(\epsilon)=\alpha_{\text {res }} \frac{(\Gamma / 2)^{2}}{\left(\epsilon-\epsilon_{\mathrm{res}}\right)^{2}+(\Gamma / 2)^{2}},
$$

which corresponds to an exponentially decaying excitation. $\Gamma$ is the full width at half maximum of the resonance and is related to the lifetime of the resonance. $\epsilon_{\text {res }}$ is the mid point of the resonance, which we will refer to as the resonance energy. The excitation probability is proportional to $\alpha_{\text {res }}$, so it is closely related to the resonance cross section.

We estimate the three parameters, $\Gamma, \epsilon_{\text {res }}$, and $\alpha_{\text {res }}$, in Eq. (4) by performing a least-squares fit of the maximum overlaps obtained by inserting Eqs. (4) and (3) in Eq. (2) to the maximum overlaps shown in Fig. 4. This gives the values of $\Gamma=1.4 \mathrm{eV}$ and $\epsilon_{\text {res }}=9.8 \mathrm{eV}$ above the Fermi level and $\alpha_{\text {res }}$ $=5.4 \times 10^{-3}$. The value of $\epsilon_{\mathrm{res}}=9.8 \mathrm{eV}$ may seem high but one should keep in mind that for this system the Fermi level lies at the highest occupied molecular orbital of the nitrogen molecule and that the surface cannot create an image charge, which would lower the resonance energy. The $9.8 \mathrm{eV}$ also seem reasonable, when comparing to the lowest excitation energies of the nitrogen molecule, which are at the same level. In Sec. III B we will calculate the equivalent number for a molecule sitting at a more realistic cluster surface. Here we do indeed find that the presence of electrons in the surface and the possibility of an image charge will lower this number.

The resulting Lorentz distribution is shown in Fig. 6, where $W(\epsilon)$ from Eq. (3) is also plotted for different momenta of the hot-electron orbital. From this it is evident that the resonance features from Fig. 4 arise because the energy distribution of the hot electron passes the resonance as the momentum of the hot electron is increased.

Figure 7 shows how close the true maximum overlaps fits with the ones obtained by inserting the optimal values of $\Gamma$, $\epsilon_{\text {res }}$, and $\alpha_{\text {res }}$ into Eqs. (2)-(4). It is seen that they agree quite well, especially it should be noted that the double-peaked feature at Wf. No. 5 is reproduced. This indicates that the assumptions made in Eqs. (2)-(4) are reasonable and that $\Gamma$, $\epsilon_{\text {res }}$, and $\alpha_{\text {res }}$ are truly properties of the system and not the arbitrarily chosen orbitals for the hot electrons.

\section{B. Nitrogen on a ruthenium cluster}

The system considered in Sec. III A is only a very crude approximation to a real system. First of all, in a real system the surface will be build from individual atoms and, perhaps more importantly, the other electrons in the surface will feel the hot electron, giving rise to a screening effect.

The intention of this section is to utilize the experiences gained in Sec. III A on a more realistic system, i.e., we want to probe the resonance with some wave function for the hot electron and then perform the fitting of Eqs. (2)-(4) in order to extract the values of $\Gamma, \epsilon_{\text {res }}$, and $\alpha_{\text {res }}$. We will consider the cluster shown in Fig. 8, which consists of 20 ruthenium atoms and a nitrogen molecule adsorbed on it.

The cluster is a simple model of a nitrogen molecule adsorbed on a close-packed (0001) ruthenium surface and includes the first three layers. We use a cluster instead of a slab 

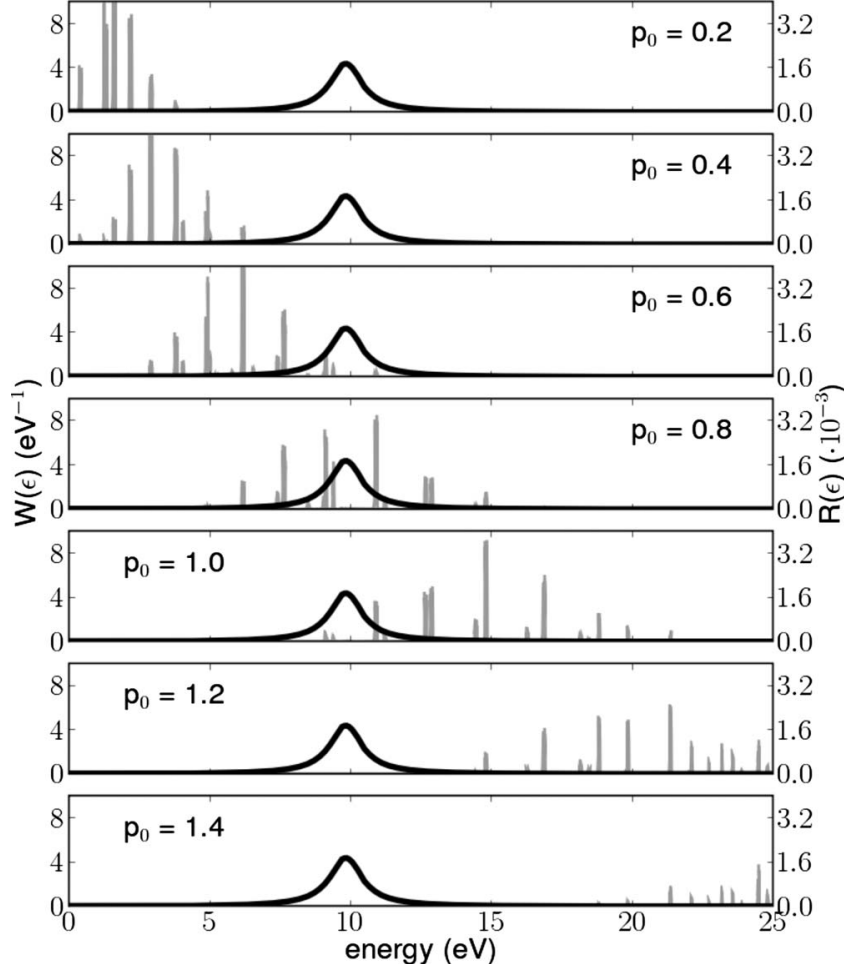

FIG. 6. The gray peaks show $W(\epsilon)$ from Eq. (3), where the delta functions have been replaced by Gaussians with a spread of $0.1 \mathrm{eV}$, for Wf. No. 1 in Table I. The different subplots are for different average momenta, $p_{0}$. The black curves show the fitted Lorentzian, $R(\epsilon)$, from Eq. (4) with the values $\Gamma=1.4 \mathrm{eV}$ and $\epsilon_{\mathrm{res}}=9.8 \mathrm{eV}$ above the Fermi level and $\alpha_{\text {res }}=5.4 \times 10^{-3}$. The left $y$ axes indicate $W(\epsilon)$ and the right $R(\epsilon)$.

in order to avoid all the difficulties that arise when applying periodic boundary conditions in a time-evolution TDDFT calculation. ${ }^{16} \mathrm{~A}$ possible intermediate system to consider

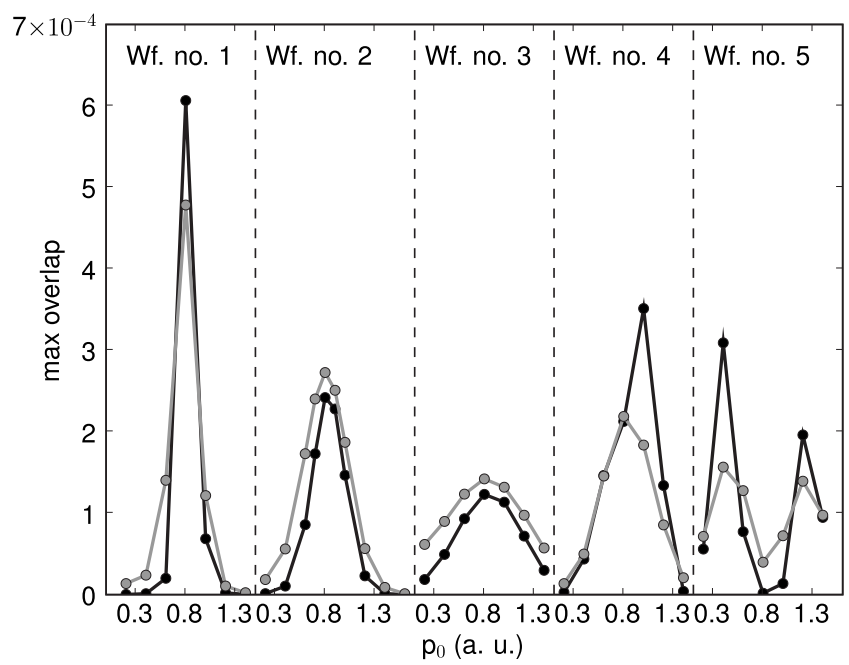

FIG. 7. The black lines show the same maximum overlaps depicted on Fig. 4. The gray lines are obtained by inserting Eqs. (3) and (4) in Eq. (2) and varying $\Gamma, \epsilon_{\text {res }}$, and $\alpha_{\text {res }}$ until the best leastsquares fit is obtained. We find this to be at $\Gamma=1.4 \mathrm{eV}$ and $\epsilon_{\text {res }}$ $=9.8 \mathrm{eV}$ above the Fermi level and $\alpha_{\text {res }}=5.4 \times 10^{-3}$.

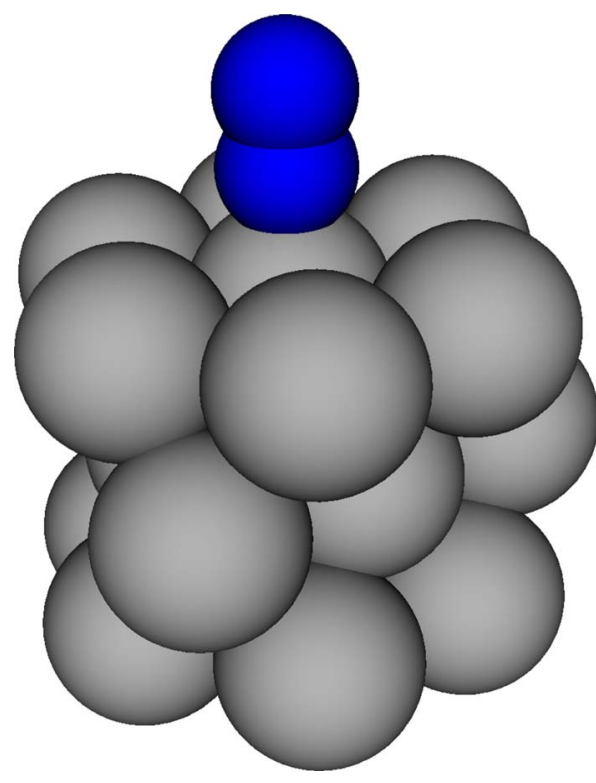

FIG. 8. (Color online) The ruthenium cluster with adsorbed nitrogen, on which we perform calculations in Sec. III B. The gray atoms are ruthenium and the dark (online: blue) nitrogen. The cluster has 20 ruthenium atoms.

would be a jellium surface, where the external step potential is made deeper and combined with extra electrons inside the surface. One would not gain much compared to the cluster calculation with respect to the calculational complexity and effort needed, so we have not done this here.

Some extra care has to put into finding a suitable starting orbital of the hot electron in this system because of the other electrons present in the cluster, which the orbital of the hot electron must be orthogonal to. Furthermore the Fourier transform of the hot-electron orbital should be quite simple, preferably with just one peak, such that we can expect that the fitting described in Sec. III A can be done easily. The procedure we choose is to first perform an ordinary groundstate DFT calculation on the 20 atoms ruthenium cluster without the nitrogen molecule. We then project the function,

$$
\Psi(r, \theta, z)=J_{1}\left(\frac{r}{r_{0}}\right) e^{i \theta} e^{-(z+3.3)^{2} / 3.3^{2}},
$$

onto the space spanned by the 116 lowest lying Kohn-Sham orbitals. $J_{1}(r)$ is a Bessel function of the first kind and $r, \theta$, and $z$ are the usual semipolar coordinates and the equation is in atomic units. $r_{0}$ is chosen such that the first node of $J_{1}\left(\frac{r}{r_{0}}\right)$ lies at $r=8 a_{0} . z=0$ corresponds to the $z$ value of the highest lying layer of atoms in the cluster. We normalize this projected version of $\Psi(r, \theta, z)$ and multiply it with $e^{i p_{0} z}$, where $p_{0}$ is the average momentum and use it as the starting orbital for the hot electron. With this choice we ensure that the Fourier transform of the starting orbital only has one significant peak, as it can be seen from Fig. 9, and we ensure that the orbital is nicely localized within the cluster. The choice of $\Psi(r, \theta, z)$ in Eq. (5) is made because it only has a single peak in the Fourier representation and the $e^{i \theta}$ factor gives it the $\Pi$ symmetry required in order for the electron to interact 


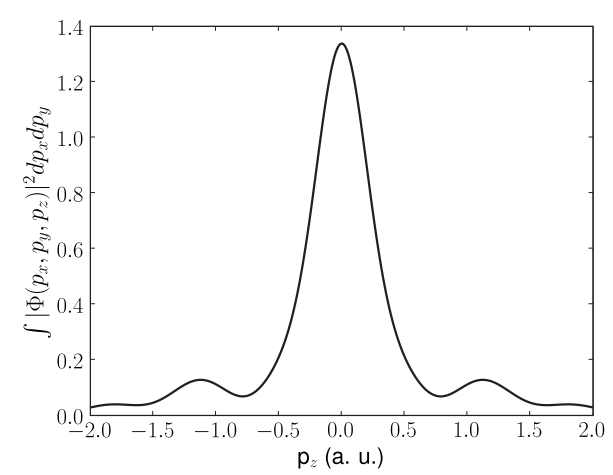

FIG. 9. The momentum space representation of the hot-electron wave function squared and integrated over the directions parallel to the surface.

with the $2 \pi^{*}$ orbitals of the nitrogen molecule. Other than that the exact choice of $\Psi(r, \theta, z)$ is not so critical. We have tried both varying the number of included KS orbitals and the parameters of the Gaussian in Eq. (5).

Finally a ground-state DFT calculation is performed on the cluster with the nitrogen molecule attached and all the occupied Kohn-Sham orbitals are othogonalized to the starting orbital of the hot electron through an ordinary GramSchmidt orthogonalization procedure. Furthermore one spin-up electron is removed at the Fermi level and placed in the orbital of the hot electron, still with spin-up. This is then used as the starting point for the time-evolution TDDFT calculation. Spins are treated using an ordinary spinpolarization procedure.

As it was also done in Sec. III A the time-evolution calculations are performed for different values of the average momentum, $p_{0}$. Figure 10 shows the maximum projection of the hot-electron orbital onto the subspace spanned by the $2 \pi^{*}$ orbitals of the nitrogen molecule within the first $3 \mathrm{fs}$ as a

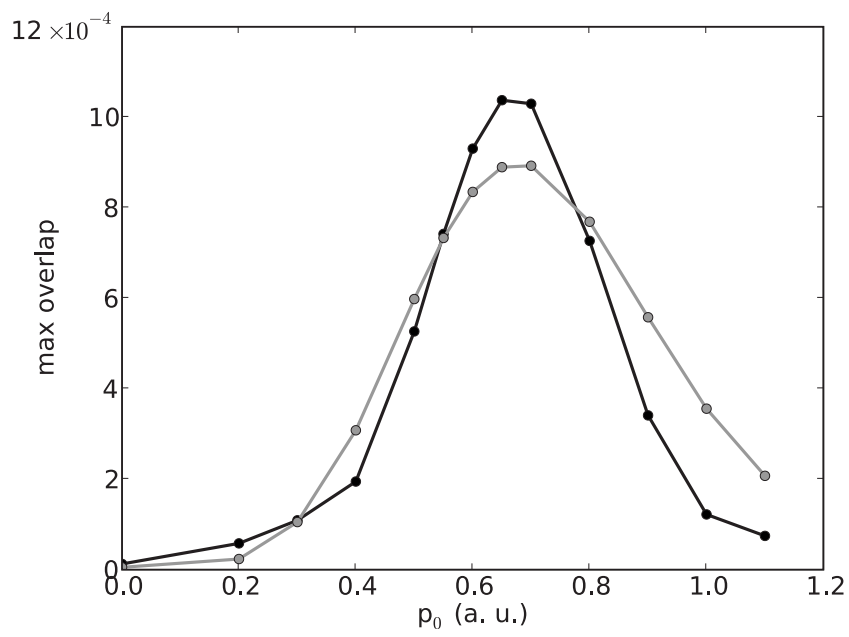

FIG. 10. The black curve shows the amount of electron that gets into the $2 \pi^{*}$ orbital of the molecule within the first three fs plotted as a function of the average momentum of the hot electron, $p_{0}$. The gray lines are obtained by inserting Eqs. (3) and (4) in Eq. (2) and varying $\Gamma, \epsilon_{\text {res }}$, and $\alpha_{\text {res }}$ until the best least-squares fit is obtained. We find this to be at $\Gamma=0.36 \mathrm{eV}, \epsilon_{\mathrm{res}}=4.9 \mathrm{eV}$, and $\alpha_{\text {res }}=1.9$ $\times 10^{-3}$.

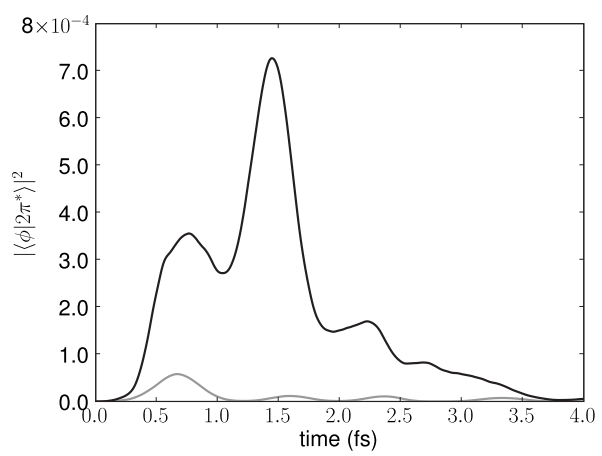

FIG. 11. The orbital of the hot electron, $\phi$, projected to the plane spanned by the two $2 \pi^{*}$ orbitals of the molecule squared as a function of time. The black curve is at a momentum of $p_{0}=0.8$ a.u. and the gray curve is at $p_{0}=0.2$.

function of $p_{0}$. Again a clear resonance peak is found. From Fig. 11 it is also seen that the difference between an electron hitting resonance and an off-resonance electron is not only the size of the overlap with the molecular orbital but also the time the electron stays there. An electron hitting resonance will stay on the molecule for some time before returning to the surface, which seems physically reasonable. This was also what we saw in Sec. III A.

Figure 10 shows also the least-squares fit we obtain by varying $\Gamma, \epsilon_{\text {res }}$, and $\alpha_{\text {res }}$ in Eqs. (2)-(4). The optimal values we find are $\Gamma=0.36 \mathrm{eV}$ and $\epsilon_{\mathrm{res}}=4.9 \mathrm{eV}$ above the Fermi energy and $\alpha_{\text {res }}=1.9 \times 10^{-2}$. The $\Gamma$ value is quite uncertain because $R(\epsilon)$ is much more localized than $W(\epsilon)$ in Eq. (2); i.e., we try to determine the shape of a very thin function by probing it with a very wide. The $4.9 \mathrm{eV}$ resonance energy is significant lower than the $9.8 \mathrm{eV}$ found in Sec. III A, which was also expected as the Fermi level is now raised by the electrons in the surface and as the resonance energy is lowered by an image charge effect with the surface. Inverse photoemission experiments for $\mathrm{N}_{2}$ on a nickel surface give an energy of approximately $4.4 \mathrm{eV}^{28}$ When we perform $\Delta \mathrm{SCF}$ calculations in the manner described in Ref. 11 we find that there is only a minor difference in energy between having a nickel and a ruthenium surface. This indicates that the value of $4.9 \mathrm{eV}$ is quite reasonable. It is also worth noticing that the projected density of states for the $2 \pi$ states has its maximum between 2 and $3 \mathrm{eV}$ above the Fermi level as we will show in Sec. IV. This means that the optimal value of $\epsilon_{\text {res }}$ cannot be explained as a mere matching in energy between the hot electron and the $2 \pi$ Kohn-Sham states. This is probably because the energy of the Kohn-Sham states will change as the density changes, which fits well with a $\triangle$ SCF picture of the situation. In Sec. IV we will consider the value of $\Gamma$.

We expect that the found $\alpha_{\text {res }}$ values will depend on the cross-section areas of the considered systems, i.e., the unitcell cross section in Sec. III A and the cluster cross section in this section since the hot-electron orbital is spread over these areas. It will probably be reasonable to assume that $\alpha_{\text {res }}$ is inversely proportional to the cross-section area of the system, i.e.,

$$
A_{\text {res }}=\alpha_{\text {res }} \times A_{\text {system }},
$$

where $A_{\text {res }}$ is the resonance cross section and $A_{\text {system }}$ is the cross section of the system. With this crude approximation 

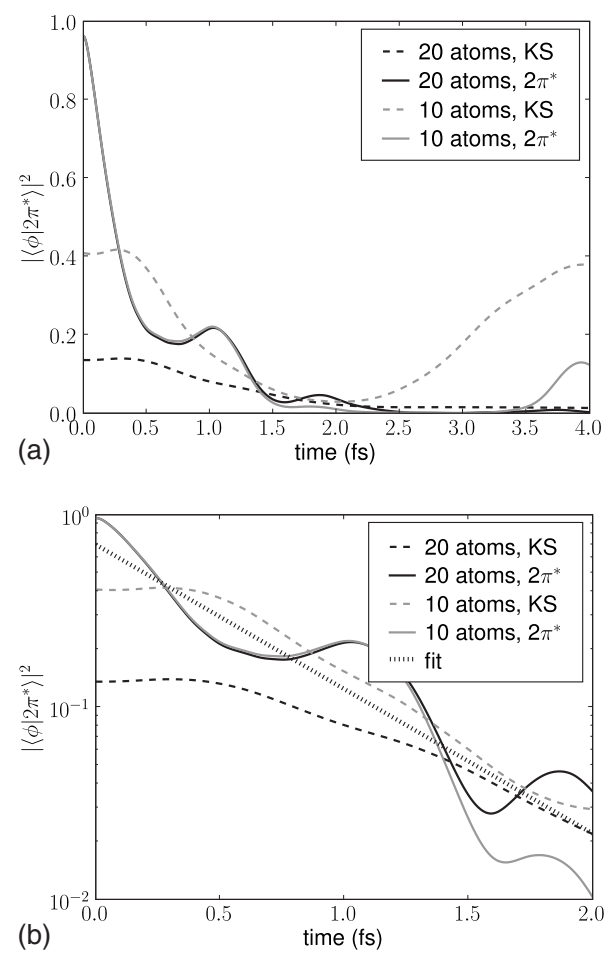

FIG. 12. The orbital of the excited electron projected plane spanned by the $2 \pi^{*}$ orbitals of the nitrogen molecule squared as a function of time for four different calculations. The only difference between upper and lower panels is that they use a normal and a logarithmic scale, respectively, on the $y$ axes. Two of the calculations are performed on the 20-atom cluster from Fig. 8 and the other two on the 10-atom cluster from Fig. 13. The difference between the two calculations on each cluster is the state of the excited electron. Either the Kohn-Sham orbital with the largest overlap with the $2 \pi^{*}$ orbitals of the molecule is used or simply one of the $2 \pi^{*}$ orbitals found from a gas phase calculation is used.

we can make a very rough estimate of the resonance cross section. As the system cross section we use the area that seven atoms fill in a ruthenium (0001) surface because there are seven atoms in the top layer of the cluster. We then get $A_{\text {res }}=0.88 \AA^{2}$. By performing calculations for different system cross sections it would be possible to test the assumption of Eq. (6). However, this is beyond the scope of this paper.

\section{DECAY OF AN EXCITATION}

In Sec. III we simulated the entire event of an electron hitting a molecular resonance and then returning to the surface. It is, however, also interesting to just consider the decay of an excited molecule, in order to gain some information on the lifetime, which is a very important parameter when trying to calculate the probability that the electron will induce some chemistry. ${ }^{12}$ Again we consider a nitrogen molecule adsorbed on a ruthenium cluster.

The decay is considered by exciting an electron to the nitrogen molecule and then monitoring the overlap between the electron and the molecular $2 \pi^{*}$ orbitals as time passes. Figure 12 shows this overlap as a function of time for four different calculations.

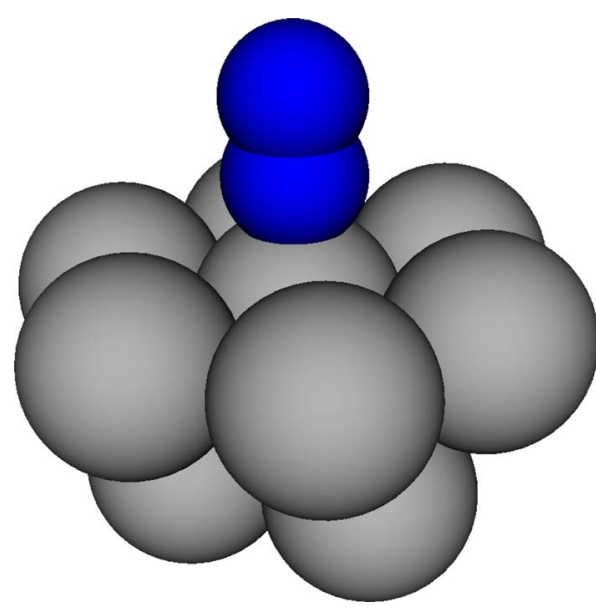

FIG. 13. (Color online) One of the ruthenium clusters used in Sec. IV. The gray atoms are ruthenium and the dark (online: blue) are an adsorbed nitrogen molecule. The cluster has 10 ruthenium atoms.

The difference between the calculations is the state the electron has started in and/or the size of the cluster considered. The electron is either started in the Kohn-Sham eigenfunction with the largest overlap with the $2 \pi^{*}$ orbitals of the nitrogen molecule or simply in one of the $2 \pi^{*}$ orbitals of the nitrogen molecule found from a gas phase calculation. The cluster is either the 20 atoms ruthenium cluster shown in Fig. 8 or the 10 atoms ruthenium cluster shown in Fig. 13.

From the semilogarithmic plot in the lower panel of Fig. 12 it is seen that the lifetime is quite similar for all four calculations, indicating that the arbitrary choice of a starting orbital for the excited electron and the size of the cluster is not too critical when estimating the lifetime. From the linear fit on the semilogarithmic plot we get a lifetime of $\tau$ $=0.6$ fs. With the use of Heisenberg's uncertainty relation, $\Delta t \Delta E \approx \hbar$, we can associate this lifetime with an uncertainty in the resonance energy: $\Delta E \approx 1.1 \mathrm{eV}$. By comparing this with the density of states projected onto the plane spanned by the $2 \pi^{*}$ orbitals of the nitrogen molecule, as it is done in Fig. 14, we see that it fits quite well with the spread of the projected density of states. This indicates that estimating excitation lifetimes from the projected density of Kohn-Sham states is reasonable. In Sec. III B we found a value of $\Gamma$ $=0.36 \mathrm{eV}$, which is approximately a factor of 3 different from the $\Delta E$ found here. This difference is consistent with the large uncertainty attached to the $\Gamma$ value determined in the fitting procedure.

From the upper panel of Fig. 12 it is also seen that the electron returns to the $2 \pi^{*}$ orbitals after a few femtoseconds for the ten-atom cluster, when the electron is placed in a Kohn-Sham orbital. This looks like a two-level oscillation and in fact a more careful analysis reveals that the electron oscillates between two Kohn-Sham orbitals, i.e., the couplings to the other Kohn-Sham orbitals are very low. Similar effects can also be seen in the other calculations if they are continued and is a consequence of the fact that it does not make sense to continue the calculations too far because the system cannot dissipate the electronic energy as it would when connected to a large surface. 


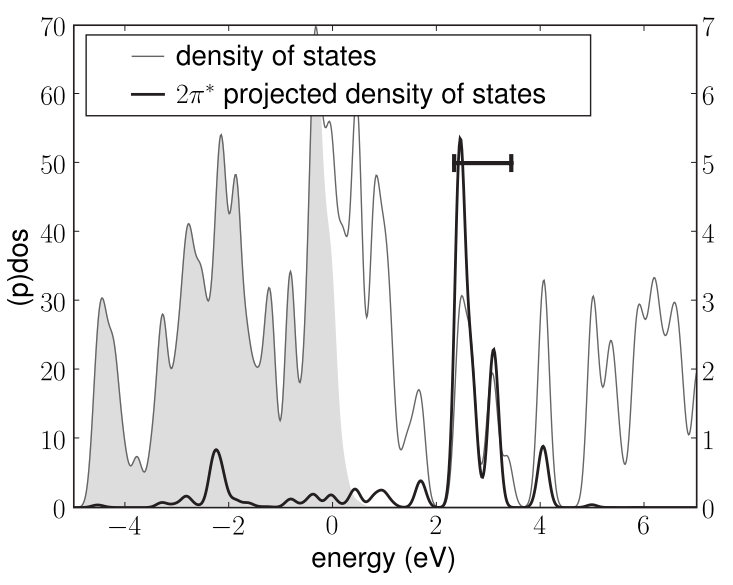

FIG. 14. The density of states for the 20 atoms cluster of Fig. 8 and the density of states projected onto the plane spanned by the $2 \pi^{*}$ orbitals of the nitrogen molecule. The inserted bar has a length of $1.1 \mathrm{eV}$, which corresponds to the expected uncertainty in energy of the $2 \pi^{*}$ resonance (see text). The light gray filling indicates the occupied states. Left axis is the density of states and the right the projected density of states.

\section{SUMMARY}

We have applied time-evolution TDDFT to model the interaction of electrons with molecular resonances at surfaces. More specifically we have considered systems consisting of a nitrogen molecule adsorbed either on a simple fictive surface or a more realistic ruthenium cluster. We found that this TDDFT approach can be used to extract several physical properties of the systems:

(1) when an electron collides with a molecule from inside the surface, some of the orbital is reflected, some places itself in a molecular state, and in some case some of it passes the molecule. This can be associated with the probabilities of reflection, excitation, and transmission.
(2) When the momentum (or energy) of the incoming electron is varied a resonance feature is observed.

(3) An electron hitting the resonance will stay on the molecule for some time in contrast to an electron hitting offresonance.

(4) We obtain reasonable values for the resonance energies. As expected the resonance energy is lowered by the contact with a realistic surface.

(5) The decay of the electronic excitation fits an exponential quite well.

Furthermore we have established a picture where the incoming hot electron can be considered as a probe, which probes the resonance. Using a fitting procedure we have been able to extract resonance properties, which apparently are system specific and not dependent on the exact nature of the incoming electron orbital. We have shown how this picture can be applied to a more realistic system consisting of a molecule adsorbed on a cluster of atoms.

Finally we compared the lifetime observed in a timeevolution TDDFT calculation with a simple projected density-of-states analysis. We found that they agree quite well.

\section{ACKNOWLEDGMENTS}

The Center for Individual Nanoparticle Functionality CINF is sponsored by the Danish National Research Foundation. This work was supported by the Danish Center for Scientific Computing. A.R. acknowledges funding by the Spanish MEC (Grant No. FIS2007-65702-C02-01), "Grupos Consolidados UPV/EHU del Gobierno Vasco" (Grant No. IT-319-07), CSIC, and European Community through e-I3 ETSF project (Project No. INFRA-2007-1.2.2; Grant Agreement Number 211956). We acknowledge support by the Barcelona Supercomputing Center, "Red Espanola de Supercomputacion," and SGIker ARINA (UPV/EHU). The Center for Atomic-Scale Materials Design CAMD is sponsored by the Lundbeck Foundation.

\footnotetext{
*schiotz@fysik.dtu.dk

${ }^{1}$ Laser Spectroscopy and Photochemistry on Metal Surfaces, edited by H.-L. Dai, and W. Ho (World Scientific, Singapore, 1995).

${ }^{2}$ J. W. Gadzuk, Phys. Rev. B 44, 13466 (1991).

${ }^{3}$ R. E. Palmer and P. J. Rous, Rev. Mod. Phys. 64, 383 (1992).

${ }^{4}$ R. R. Cavanagh, D. S. King, J. C. Stephenson, and T. F. Heinz, J. Phys. Chem. 97, 786 (1993).

${ }^{5}$ X.-Y. Zhu, Annu. Rev. Phys. Chem. 45, 113 (1994).

${ }^{6}$ D. G. Busch and W. Ho, Phys. Rev. Lett. 77, 1338 (1996).

${ }^{7}$ J. W. Gadzuk, Phys. Rev. Lett. 76, 4234 (1996).

${ }^{8}$ M. Bonn, S. Funk, C. Hess, D. N. Denzler, C. Stampfl, M. Scheffler, M. Wolf, and G. Ertl, Science 285, 1042 (1999).

${ }^{9}$ J. A. Misewich, T. F. Heinz, and D. M. Newns, Phys. Rev. Lett. 68, 3737 (1992).

${ }^{10}$ L. B. Thomsen, G. Nielsen, S. B. Vendelbo, M. Johansson, O. Hansen, and I. Chorkendorff, Phys. Rev. B 76, 155315 (2007).

${ }^{11}$ J. Gavnholt, T. Olsen, M. Engelund, and J. Schiøtz, Phys. Rev. B 78, 075441 (2008).
}

${ }^{12}$ T. Olsen, J. Gavnholt, and J. Schiøtz, Phys. Rev. B 79, 035403 (2009).

${ }^{13}$ T. Kreibich and E. K. U. Gross, Phys. Rev. Lett. 86, 2984 (2001).

${ }^{14}$ R. Baer and N. Siam, J. Chem. Phys. 121, 6341 (2004).

${ }^{15}$ OCTOPUS is a freely available real-space-time-dependent density functional theory code (see www.tddft.org/programs/octopus/).

${ }^{16}$ A. Castro, H. Appel, M. Oliveira, C. Rozzi, X. Andrade, F. Lorenzen, M. Marques, E. Gross, and A. Rubio, Phys. Status Solidi B 243, 2465 (2006)

${ }^{17}$ A. Zangwill and P. Soven, Phys. Rev. A 21, 1561 (1980).

${ }^{18}$ Pseudopotentials from the Fritz-Haber institute are available on the website www.abinit.org/Psps/?text=./Psps/LDA_FHI/fhi

${ }^{19}$ N. Troullier and J. L. Martins, Phys. Rev. B 43, 1993 (1991).

${ }^{20}$ M. Hochbruck and C. Lubich, SIAM (Soc. Ind. Appl. Math.) J. Numer. Anal. 41, 945 (2003).

${ }^{21}$ M. Hochbruck and C. Lubich, SIAM (Soc. Ind. Appl. Math.) J. Numer. Anal. 34, 1911 (1997).

${ }^{22}$ D. M. Ceperley and B. J. Alder, Phys. Rev. Lett. 45, 566 (1980). 
${ }^{23}$ C. G. Broyden, Math. Comput. 19, 577 (1965).

${ }^{24}$ The GPAW code including the implementation of $\triangle \mathrm{SCF}$ is available as a part of the CAMPOS software: www.camp.dtu.dk/ Software

${ }^{25}$ J. J. Mortensen, L. B. Hansen, and K. W. Jacobsen, Phys. Rev. B
71, 035109 (2005).

${ }^{26}$ Lengths in Bohr radii and energies in Hartree.

${ }^{27}$ K. S. Thygesen, Phys. Rev. Lett. 100, 166804 (2008).

${ }^{28}$ P. D. Johnson and S. L. Hulbert, Phys. Rev. B 35, 9427 (1987). 